\title{
Multidrug resistance in Pseudomonas aeruginosa isolated from nosocomial respiratory and urinary infections in Aleppo, Syria
}

\author{
Maysa Mahfoud ${ }^{1}$, Mona Al Najjar², Abdul Rezzak Hamzeh ${ }^{1}$ \\ ${ }^{1}$ Department of Biochemistry and Microbiology, Faculty of Pharmacy, University of Aleppo, Aleppo, Syria \\ ${ }^{2}$ Centralized Clinical Microbiological Laboratory in Albasel and Ibn Rushd Hospitals, Aleppo, Syria
}

\begin{abstract}
Introduction: Pseudomonas aeruginosa represents a serious clinical challenge due to its frequent involvement in nosocomial infections and its tendency towards multidrug resistance.

Methodology: This study uncovered antibiotic susceptibility patterns in 177 isolates from inpatients in three key hospitals in Aleppo, the largest city in Syria.

Results: Exceptionally low susceptibility to most routinely used antibiotics was uncovered; resistance to ciprofloxacin and gentamicin was $64.9 \%$ and $70.3 \%$, respectively. Contrarily, susceptibility to colistin was the highest $(89.1 \%)$.

Conclusions: Multidrug resistance was rife, found at a rate of $53.67 \%$ among studied $P$. aeruginosa isolates.
\end{abstract}

Key words: Pseudomonas aeruginosa; nosocomial infections; multidrug resistance; respiratory infections; urinary infections; Syria.

J Infect Dev Ctries 2015; 9(2):210-213. doi:10.3855/jidc.5643

(Received 22 July 2014 - Accepted 15 October 2014)

Copyright (C) 2015 Mahfoud et al. This is an open-access article distributed under the Creative Commons Attribution License, which permits unrestricted use, distribution, and reproduction in any medium, provided the original work is properly cited.

\section{Introduction}

Pseudomonas aeruginosa is a Gram-negative, obligate aerobic, and oxidase-positive bacillus. In healthy subjects, $P$. aeruginosa is part of skin flora; however, it can cause serious morbidity in individuals with predisposing factors such as considerable body injury or certain pulmonary conditions (e.g., cystic fibrosis) [1]. This opportunistic pathogen is a common cause of hospital-acquired infections and, to a lesser extent, community-acquired infections. Examples of infections include pneumonia, urinary tract infections (UTIs), bacteremia, and wound and burn infections. Nosocomial pneumonia due to $P$. aeruginosa is usually associated with poor survival rates $[2,3]$.

Despite rapid advancement in healthcare provision, partially through the introduction of highly effective antibiotics into clinical practice, $P$. aeruginosa remains a pathogen to be reckoned with. This is certainly the case at intensive care units (ICUs), particularly in patients who are put on a mechanical ventilator [3]. Nosocomial bacteremia accounts for over $50 \%$ of ICU infections, and the causative pathogen is frequently found to be $P$. aeruginosa [4].

Multidrug resistance (MDR) displayed by $P$. aeruginosa is usually found during empirical therapy or following lengthy exposure to antibiotics. $P$. aeruginosa has myriad intrinsic and extrinsic factors that work together to cause resistance to structurally and functionally dissimilar antibiotics. Certain resistance mechanisms that have been found in other bacteria may also play a role in MDR in $P$. aeruginosa [5]. Importantly, $P$. aeruginosa can express chromosomally encoded multidrug efflux pump genes coupled with outer membrane impermeability. $P$. aeruginosa possesses a spectrum of virulence factors, including a type III secretion system, which contributes massively to its pathogenicity [2].

Additionally, $P$. aeruginosa is capable of developing acquired antimicrobial resistance. The latter emerges due to mutations arising in chromosomally-encoded genes and/or horizontal gene transfer of resistance genes, a variety of which can be carried together on transferable structures (integrons) [6]. Finally, $P$. aeruginosa is adept at forming biofilms on surfaces, so it can survive antibiotics and other disinfectant chemicals as well as the body's innate and adaptive inflammatory defenses [2,7].

Studies from different geographical localities worldwide highlight the dilemma of increased occurrence of infections with MDR $P$. aeruginosa. These pose a heavy human and economical burden 
because antibiotics are costly and frequently ineffective, leading to high mortality rates [2-7]. Informative data about these types of infections are lacking from Syria; therefore, this study was conducted to probe for prevalence rates of MDR among $P$. aeruginosa isolates in northern Syria. This study focused on susceptibility towards antimicrobial agents that are routinely administered in local hospitals.

\section{Methodology}

This cross-sectional study was conducted between September 2011 and September 2012 at three major hospitals in Aleppo, Syria: Ibn Rushd Hospital, Aleppo University Hospital, and Al-Basel Center for Heart Disease and Cardiac Surgery. The relevant approval was obtained from the ethics committee of Aleppo University. Samples were taken from ICU patients with lower respiratory infections (LRIs) and from patients with nosocomial UTIs. The mean age of the patients was 52.7 years. All lower respiratory and urinary samples came from inpatients who had been hospitalized for $\geq 48$ hours. A total of 177 nonrepetitive isolates of $P$. aeruginosa were found, and the guidelines of the Clinical and Laboratory Standards Institute (CLSI) were followed for pathogen identification and testing [8]. Female patients contributed 59 of the isolates, while the rest came from male patients. $P$. aeruginosa was isolated and identified using standard biochemical reactions, and the results were confirmed using the Phoenix Automated Microbiology System by BD (Becton, Dickinson and Company, Franklin Lakes, USA). Afterwards, bacterial isolates were tested for susceptibility to antibiotics by the standard KirbyBauer disk diffusion method. Twenty-three antimicrobial susceptibility testing disks (Oxoid, Basingstoke, UK; codes are listed in Table 1) were used, and results were determined and interpreted based on CLSI guidelines. Multidrug resistance was defined as resistance to three or more unrelated antibacterial agents.

\section{Results}

This study sought to characterize the pattern of antibiotic resistance among $P$. aeruginosa isolates obtained from three major hospitals in the largest urban center in Aleppo, northern Syria. A total of 138 isolates were obtained from ICU patients with LRIs, and the rest (39 isolates) came from patients with UTIs. Around a quarter (26\%) of the LRIs isolates and over half $(59 \%)$ of the UTIs isolates came from

Table 1. Antibiogram depicting antimicrobial susceptibility of Pseudomonas aeruginosa isolates collected between September 2011 and September 2012 in Aleppo, Syria

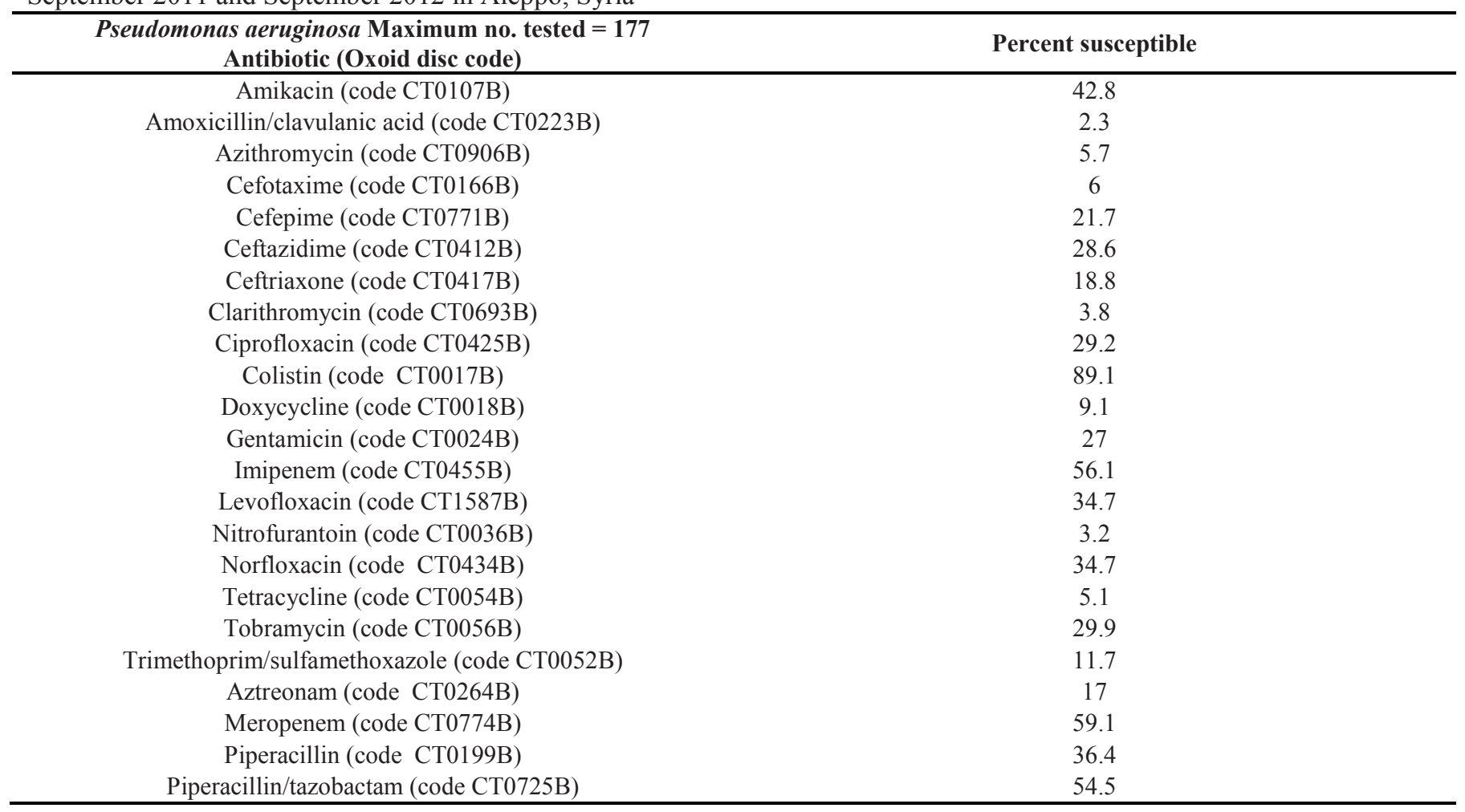


women.

Generally, tested $P$. aeruginosa isolates displayed high levels of antibiotic resistance to the majority of antibiotics that are routinely used in local clinical settings (Table 1). Levels of susceptibility to amoxicillin/clavulanic acid and nitrofurantoin were the lowest: $2.3 \%$ and $3.2 \%$, respectively. Susceptibility to each of clarithromycin, tetracycline, azithromycin, cefotaxime, and doxycycline never exceeded $10 \%$. Considerably higher susceptibility was obtained with imipenem $(56.1 \%)$ and meropenem (59.1\%); however, the most optimal response in vitro was obtained with colistin (89.1\%).

\section{Discussion}

To put our results in the right context, a comprehensive multicenter study by Sader et al. [9] was used as a major referential point. The latter study covered 31 medical centers located in 13 European countries plus Turkey and Israel, and it reported on antibiotic resistance and MDR of $P$. aeruginosa. Generally, susceptibility rates uncovered in this study were alarmingly lower than these reported by Sader et al. [9]. Examples include the fourth-generation cephalosporin, cefepime $(21.7 \%$ in Syria versus $71.4 \%$ in the 15 countries covered in [9]), the fluoroquinolone, levofloxacin $(34.7 \%$ in Syria versus $64.1 \%$ in the 15 countries covered in [9]), and piperacillin/tazobactam. Additionally, resistance to ciprofloxacin was as high as $64.9 \%$. The latter antibiotics are misused in Syria widely and frequently due to their low prices and the ease with which the general public can obtain them without a prescription.

Resistance to gentamicin $(70.3 \%)$ was much higher than to amikacin (40\%). It is worth noting that the latter antibiotic is used less frequently in local healthcare establishments, mainly due to its overly publicized ototoxicity and nephrotoxicity. Also, amikacin is far less vulnerable to the destructive effects of aminoglycoside-modifying enzymes produced by $P$. aeruginosa. Nevertheless, the Syrian figures are much higher than their counterparts from Europe, Turkey, and Israel [9], where resistance rates to gentamicin and amikacin were found to be $22 \%$ and $11.4 \%$, respectively.

Nearly 54\% (95 isolates) of tested $P$. aeruginosa isolates were MDR. Reported rates of MDR among $P$. aeruginosa varied widely based on sample size, source of samples, and geographical locality. For instance, numerous studies from Iran reported on multidrugresistant $P$. aeruginosa. One of the larger studies [10] reported an extremely low rate $(5.46 \%)$. A higher rate
(31.9\%) was reported by Sader et al. [9] from two Middle Eastern countries and 13 European countries. Still, the latter rate is significantly lower than the figure uncovered in this study.

There have been many reports worldwide describing the trend of increasing antibiotic resistance in general and MDR in particular among $P$. aeruginosa [11,12]. For example, a nine-year American study showed a considerable increase in multidrug-resistant $P$. aeruginosa, from 1\% in 1994 to $16 \%$ in 2002 . This is a good indicator of the degree of increase in MDR in the largest industrialized country in the world [13]. On the bright side, reviving some old antibiotics might offer an alternative way to fight MDR [14]. Our results are necessary for guiding local health authorities in Syria; however, on a global scale, our findings help to fill a significant information gap by demonstrating such a high rate of antibacterial resistance. Our data is expected to encourage correct use of antibiotics and to persuade practitioners to rely more on antibiotic susceptibility tests in making treatment decisions in individual cases.

\section{Acknowledgements}

We would like to thank the University of Aleppo, Syria, for funding the work that led to this publication.

\section{References}

1. O'Sullivan BP1, Freedman SD (2009) Cystic fibrosis. Lancet 373: 1891-1904. doi: 10.1016/S0140-6736(09)60327-5.

2. Gillespie S, Hawkey PM (2006) Principles and Practice of Clinical Bacteriology, Second Edition, Chichester, UK: Wiley. 620 p.

3. Zavascki AP, Barth AL, Fernandes JF, Moro AL, Gonçalves AL, Goldani LZ (2006) Reappraisal of Pseudomonas aeruginosa hospital-acquired pneumonia mortality in the era of metallo-beta-lactamase-mediated multidrug resistance: a prospective observational study. Crit Care 10: R114.

4. Vitkauskienè A, Skrodenienè E, Dambrauskienė A, Macas A, Sakalauskas R (2010) Pseudomonas aeruginosa bacteremia: resistance to antibiotics, risk factors, and patient mortality. Medicina (Kaunas) 46: 490-495.

5. Mosel M, Li L, Drlica K, Zhao X (2013) Superoxidemediated protection of Escherichia coli from antimicrobials. Antimicrob Agents Chemother 57: 5755-5759. doi: 10.1128/AAC.00754-13.

6. Strateva T, Yordanov D (2009) Pseudomonas aeruginosa - a phenomenon of bacterial resistance. J Med Microbiol 58: 1133-1148. doi: 10.1099/jmm.0.009142-0.

7. Qiu X, Kulasekara BR, Lory S (2009) Role of Horizontal Gene Transfer in the Evolution of Pseudomonas aeruginosa Virulence. Genome Dyn 6: 126-139. doi: $10.1159 / 000235767$.

8. Clinical and Laboratory Standards Institute (2005) Performance standards for antimicrobial susceptibility testing. NCCLS documents M100-S12. CLSI: Wayne, PA. 
9. Sader HS, Farrell DJ, Castanheira M, Flamm RK, Jones RN (2014) Antimicrobial activity of ceftolozane/tazobactam tested against Pseudomonas aeruginosa and Enterobacteriaceae with various resistance patterns isolated in European hospitals (2011-12). J Antimicrob Chemother 69: 2713-2722.

10. Shahcheraghi F, Badmasti F, Feizabadi MM (2014) Molecular characterization of class 1 integrons in MDR Pseudomonas aeruginosa isolated from clinical settings in Iran, Tehran. FEMS Immunol Med Microbiol 58: 421-425. doi: 10.1111/j.1574-695X.2009.00636.x.

11. Vojtová V, Kolár M, Hricová K, Uvízl R, Neiser J, Blahut L, Urbánek K (2011) Antibiotic utilization and Pseudomonas aeruginosa resistance in intensive care units. New Microbiol 34: 291-298.

12. Van Eldere J (2003) Multicentre surveillance of Pseudomonas aeruginosa susceptibility patterns in nosocomial infections. $\mathrm{J}$ Antimicrob Chemother 51: 347-352.
13. D'Agata EM (2004) Rapidly rising prevalence of nosocomial multidrug-resistant, Gram-negative bacilli: a 9-year surveillance study. Infect Control Hosp Epidemiol 25: 842846.

14. Malik M, Li L, Zhao X, Kerns RJ, Berger JM, Drlica K (2014) Lethal synergy involving bicyclomycin: an approach for reviving old antibiotics. J Antimicrob Chemother 69: 3227-3235.

\section{Corresponding author}

Dr.Abdul Rezzak Hamzeh

Faculty of Pharmacy, University of Aleppo

Jamea Street, Aleppo, Syria

Phone: + 00963212633138

Fax: + 00963212633138

Email: abdul77rh@yahoo.co.uk

Conflict of interests: No conflict of interests is declared. 